\title{
$\mathrm{BaTiO}_{3}$ 纳米线的制备及其复合物介电和储能性能研究
}

\author{
王 璐 ${ }^{1}$, 孔文杰 ${ }^{2}$, 罗 行 $^{1}$, 周学凡 ${ }^{1}$, 周科朝 ${ }^{1}$, 张 ${ }^{1}$ \\ (中南大学 1. 粉末冶金国家重点实验室; 2. 材料科学与工程学院, 长沙 410083)
}

摘 要: 采用两步水热法合成钛酸钡 $\left(\mathrm{BaTiO}_{3}\right)$ 纳米线, 并以此为填充物, 聚偏氟乙烯六氟丙烯 $(\mathrm{P}(\mathrm{VDF}-\mathrm{HFP}))$ 为聚合 物基体制备介电复合物, 研究不同含量 $\mathrm{BaTiO}_{3}$ 纳米线对复合物的介电及储能性能的影响。采用 $\mathrm{X}$ 射线衍射仪、扫 描电镜、透射电镜、阻抗分析仪和铁电工作站等表征 $\mathrm{BaTiO}_{3}$ 纳米线及其复合物的物相、微观结构、介电和储能性 能。结果表明: $\mathrm{BaTiO}_{3}$ 纳米线具有典型的四方相, 且在聚合物基体中具有良好的分散性与相容性。相同频率下, 复 合物的介电常数随着 $\mathrm{BaTiO}_{3}$ 纳米线含量的增加而增加。含量为 $20 \mathrm{vol} \%$ 的复合物, 在 $1 \mathrm{kHz}$ 频率下其介电常数取得 最 大值 30.69。含量为 $5 \mathrm{vol} \%$ 的复合物, 在场强为 $240 \mathrm{kV} / \mathrm{mm}$ 时, 获得了最大的储能密度与放电能量密度, 分别 为 4.89 和 $2.58 \mathrm{~J} / \mathrm{cm}^{3}$ 。

关 键 词: 钣酸钡; 介电复合物; 介电常数; 能量密度

中图分类号: TB34 文献标识码: A

\section{Dielectric and Energy Storage Property of Dielectric Nanocomposites with $\mathrm{BaTiO}_{3}$ Nanofibers}

\author{
WANG Lu ${ }^{1}$, KONG Wen-Jie ${ }^{2}$, LUO Hang ${ }^{1}$, ZHOU Xue-Fan ${ }^{1}$, ZHOU Ke-Chao ${ }^{1}$, ZHANG Dou ${ }^{1}$
}

(1. State Key Laboratory of Powder Metallurgy, Central South University, Changsha 410083, China; 2. School of Materials Science and Engineering, Central South University, Changsha 410083, China)

\begin{abstract}
In this study, $\mathrm{BaTiO}_{3}$ nanofibers were synthesized by a two-step hydrothermal method and subsequently incorporated into poly(vinylidene fluoride-co-hexafluoropropylene) (P(VDF-HFP)) matrix to prepare nanocomposites for energy storage application. The crystalline phase, morphology and microstructure of $\mathrm{BaTiO}_{3}$ nanofibers were observed by X-ray diffraction, scanning electron microscopy and transmission electron microscopy, respectively. The dielectric properties and energy storage performance of the nanocomposites were characterized by dielectric and ferroelectric analyzer. The $\mathrm{BaTiO}_{3}$ nanofibers with tetragonal phase structure exhibited high aspect ratios, good dispersibility and compatibility in polymer matrix. The effects of volume fraction of $\mathrm{BaTiO}_{3}$ nanofibers on the dielectric constant, breakdown strength and discharged energy density of the nanocomposites were investigated systematically. The dielectric constant of the $\mathrm{BaTiO}_{3}-\mathrm{P}(\mathrm{VDF}-\mathrm{HFP})$ nanocomposites remarkably improved with the increase of $\mathrm{BaTiO}_{3}$ nanofiber contents at the same frequency. At $1 \mathrm{kHz}$, the maximum dielectric constant of the composite with $20 \mathrm{vol} \% \mathrm{BaTiO}_{3}$ nanofibers is up to 30.69 . The composite with $5 \mathrm{vol} \% \mathrm{BaTiO}_{3}$ nanofibers achieves the maximum energy storage density $\left(4.89 \mathrm{~J} / \mathrm{cm}^{3}\right)$ and discharged energy density $\left(2.58 \mathrm{~J} / \mathrm{cm}^{3}\right)$ at $240 \mathrm{kV} / \mathrm{mm}$.
\end{abstract}

Key words: $\mathrm{BaTiO}_{3}$; dielectric nanocomposite; dielectric constant; energy density

收稿日期：2018-01-29; 收到修改稿日期：2018-04-14

基金项目: 国家自然科学基金(51672311); 湖南省科技项目(2016WK2022); 中南大学博士后基金(140050006)

National Natural Science Foundation of China (51672311); Science and Technology Project of Hunan Province, China (2016WK2022); Postdoctoral Research Foundation of Central South University (140050006)

作者简介: 王 璐(1995-), 女, 硕士研究生. E-mail: znwanglu@163.com

通讯作者: 张 斗, 教授.E-mail: dzhang@csu.edu.cn; 罗 行, 副教授. E-mail: hangluo@csu.edu.cn 
高性能的介电材料在可再生能源系统和电子设 备等领域具有广泛的应用前景, 因而备受关注, 比 如电容器、激光枪、雷达和心脏起搏器等 ${ }^{[1-5]}$ 。当前 介电材料普遍存在储能密度低的缺点, 从而导致采 用该类材料制备的可再生能源系统和电子设备体积 笨重且价格昂贵 ${ }^{[6-8]}$ 。如何提高材料的储能密度同时 降低其成本及体积成为目前研究的一大热点。研究 表明, 介电材料的储能密度主要取决于材料的介电 常数及其抗击穿电场大小 ${ }^{[9-11]}$ 。

聚合物基介电复合材料由于结合了聚合物和填 料各自的优点, 如高抗击穿电场、柔性和高介电常 数等, 而被大量研究 ${ }^{[12-15]}$ 。目前, 制备聚合物基复 合介电材料的主要途径是通过向聚合物基体中添加 导电填料构建逾渗体系或添加具有高介电常数的陶 瓷填料 ${ }^{[9,16]}$ 。钛酸钡 $\left(\mathrm{BaTiO}_{3}\right)$ 陶瓷是最典型的铁电体 陶瓷材料之一, 具有较高介电常数 $(>1000)$ 和较低 的介电损耗, 因而常选其作为介电复合材料的填充 相 $\left.{ }^{[13}, 17-18\right]$ 。聚偏氟乙烯 (PVDF) 及其共聚物, 如 P(VDF-HFP) 和 P(VDF-TrFE) 等均表现出相对较高 的介电常数 $(>10)$, 相对于其他的聚合物, 极大地降 低了与陶瓷填料之间的介电不匹配现象 ${ }^{[15]}$ 。同时, 其在电场作用下具有较强的自发极化, 使其具有明 显的铁电特性 ${ }^{[16]}$ 。因此, 这一类具有高介电常数的 含氟聚合物材料适合用于制备高性能的介电复合材 料。研究表明, 铁电陶瓷的形貌对复合材料的介电 性能和储能特性均具有重要的影响, 对于 1-3 型陶 瓷-聚合物纳米复合材料(在三维聚合物基体中添加 一维陶瓷纳米线)来说, 高长径比陶瓷纳米线比球 形陶瓷填料更能有效地提高纳米复合材料的介电性 能和能量密度 ${ }^{[19-21]}$ 。

基于上述讨论，本文采用两步水热法合成高长 径比的 $\mathrm{BaTiO}_{3}$ 纳米线, 并以此为填充物, $\mathrm{P}(\mathrm{VDF}-$ $\mathrm{HFP}$ ) 为聚合物基体, 采用流延法制备 $\mathrm{BaTiO}_{3}-\mathrm{P}$ (VDF-HFP)介电复合材料。两者的混合溶液通过长 时间超声和摚拌, 使 $\mathrm{BaTiO}_{3}$ 纳米线在基体中均匀分 散, 再通过热压消除复合材料中的孔洞裂纹等缺 陷。研究结果表明, 加入 $\mathrm{BaTiO}_{3}$ 纳米线后, 复合物 的介电常数和储能密度得到显著提高。本研究加快 了介电复合材料实际应用的进程。

\section{1 实验方法}

\section{1 实验原料}

以分析纯 $\mathrm{TiO}_{2}$ 纳米粉(99\%)、片状 $\mathrm{NaOH}(99 \%)$ 、 $\mathrm{HCl}(37 \mathrm{wt} \%)$ 以及 $\mathrm{Ba}(\mathrm{OH})_{2} \cdot 8 \mathrm{H}_{2} \mathrm{O}(99 \%)$ 作为原料制 备 $\mathrm{BaTiO}_{3}$ 纳米线; 以分析纯丙酮 $(99 \%) 、 N, N-$ 二甲
基甲酰胺( DMF，99\%)以及 $\mathrm{P}(\mathrm{VDF}-\mathrm{HFP})$ 为原料配置 $8 \mathrm{wt} \%$ 的 P(VDF-HFP)溶液。

\section{$1.2 \mathrm{BaTiO}_{3}$ 纳米线的合成}

(1) $\mathrm{Na}_{2} \mathrm{Ti}_{3} \mathrm{O}_{7}$ 的制备: 将 $1.446 \mathrm{~g} \mathrm{TiO}_{2}$ 分散至 $70 \mathrm{ml}$ 的 $10 \mathrm{~mol} \cdot \mathrm{L}^{-1} \mathrm{NaOH}$ 溶液中, 搅拌 $1 \mathrm{~h}$, 置于 $100 \mathrm{~mL}$ 高压釜中, 在 $210^{\circ} \mathrm{C}$ 下保持 $24 \mathrm{~h}$ 。收集所得 产物, 分别用去离子水和无水乙醇清洗后, 于 $60^{\circ} \mathrm{C}$ 下干燥 $12 \mathrm{~h}$ 。

(2) $\mathrm{H}_{2} \mathrm{Ti}_{3} \mathrm{O}_{7}$ 的制备: 将步骤 (1) 所得产物于 $0.2 \mathrm{~mol} \cdot \mathrm{L}^{-1}$ 的稀盐酸中浸泡 $4 \mathrm{~h}$ 后, 用去离子水和无 水乙醇清洗, 直至 $\mathrm{pH}$ 为 7 , 收集产物于 $60^{\circ} \mathrm{C}$ 下干燥 $12 \mathrm{~h}$ 。

(3) $\mathrm{BaTiO}_{3}$ 的制备: 称取 $0.15 \mathrm{~g}$ 步骤(2)的产物 加入至新制的 $0.05 \mathrm{~mol} \cdot \mathrm{L}^{-1} \mathrm{Ba}(\mathrm{OH})_{2}$ 溶液 中 (Ba: $\mathrm{Ti}=2: 1, \mathrm{at} / \mathrm{at}$ ), 超声分散 $5 \mathrm{~min}$ 后于 $100 \mathrm{~mL}$ 高 压釜中 $210^{\circ} \mathrm{C}$ 保持 $24 \mathrm{~h}$ 。所得产物用去离子水和无 水乙醇清洗后于 $60^{\circ} \mathrm{C}$ 下干燥 $12 \mathrm{~h}$, 得到最终产物。

\section{$1.3 \mathrm{BaTiO}_{3}$ 纳米线与 $\mathrm{P}(\mathrm{VDF}-\mathrm{HFP})$ 复合物的 制备}

称取 $8 \mathrm{~g} \mathrm{P}(\mathrm{VDF}-\mathrm{HFP})$ 聚合物颗粒，加至 $92 \mathrm{~g}$ 配 好的混合溶剂中 (丙酮: $\mathrm{DMF}=7: 3, V / V), 60^{\circ} \mathrm{C}$ 搅拌 待其完全溶解，配制成 $8 \mathrm{wt} \%$ 的 P(VDF-HFP)溶液。 称取适量 $\mathrm{BaTiO}_{3}$ 纳米线加入至 $\mathrm{P}(\mathrm{VDF}-\mathrm{HFP})$ 溶液中, 分别配制 $\mathrm{BaTiO}_{3}$ 纳米线含量为 $5 \mathrm{vol} \% 、 10 \mathrm{vol} \%$ 和 $20 \mathrm{vol} \%$ 的混合溶液，摚拌 $24 \mathrm{~h}$ 。然后将搅拌好的悬 浮液分别在玻璃板上浇注流延, $80^{\circ} \mathrm{C}$ 下干燥成膜, 利用平板硫化机将得到的复合物膜在 $200^{\circ} \mathrm{C} 、 15 \mathrm{MPa}$ 条件下热压消除孔洞裂纹等缺陷，膜厚度 $20 \mu \mathrm{m}$ 。

设计圆形孔直径为 $2 \mathrm{~mm}$, 孔圆心间距为 $4 \mathrm{~mm}$ 的金属掩模板, 金属掩模板是边长为 $30 \mathrm{~mm}$ 的正方 形。将压制好的复合物膜夹于 2 片金属掩模板中，上 下表面对称溅射金电极，溅射时间均为 $10 \mathrm{~min}$, 以保证金电极具有足够的厚度。作为对比，采用类 似方法制备纯 P(VDF-HFP) 膜, 溅射电极以测试电 性能。

\section{4 分析与测试}

采用扫描电镜(JSM-6390)和 FEI 公司的 Titan G2 60-300 型透射电镜对 $\mathrm{BaTiO}_{3}$ 纳米线的晶体结 构、形貌以及复合物膜的表面形貌进行表征; 采用 XRD (Rigaku D-Max/2550 $\mathrm{VB}^{+}$) 对 $\mathrm{BaTiO}_{3}$ 纳米线的 物相进行表征, 辐射波长为 $\lambda=0.15418 \mathrm{~nm}, 2 \theta=$ $5^{\circ} \sim 80^{\circ}$; 介电性能测试先在样品上下表面溅射金电 极, 测试设备为美国安捷伦公司的 $4294 \mathrm{~A}$ 阻抗分析 仪, 测试频率范围为 $40 \sim 10^{7} \mathrm{~Hz}$; 在室温和频率为 $10 \mathrm{~Hz}$ 的条件下, 采用 TF Analyzer $2000 \mathrm{FE}$ 型铁电 
工作站测试电位移-电场曲线(D-E loops) 以及抗击 穿电场。

\section{2 结果与讨论}

\section{1 $\mathrm{BaTiO}_{3}$ 纳米线结构表征}

本文采用两步水热法先制得 $\mathrm{Na}_{2} \mathrm{Ti}_{3} \mathrm{O}_{7}$ 纳米线, 通过质子交换得到 $\mathrm{H}_{2} \mathrm{Ti}_{3} \mathrm{O}_{7}$ 纳米线, 最终经过第二 步水热反应得到 $\mathrm{BaTiO}_{3}$ 纳米线, 制得的 $\mathrm{Na}_{2} \mathrm{Ti}_{3} \mathrm{O}_{7}$ 纳米线与 $\mathrm{H}_{2} \mathrm{Ti}_{3} \mathrm{O}_{7}$ 纳米线长径比较大, 半径分布范 围较窄，长度近似于 $5 \mu \mathrm{m}$ ，宽度约为 $100 \mathrm{~nm}$, 表面 光滑且分布均匀。本节采用 SEM、XRD、TEM 对 $\mathrm{BaTiO}_{3}$ 纳米线进行表征。如下图 1 所示, 其中 (a)为 $\mathrm{BaTiO}_{3}$ 纳米线的 $\mathrm{SEM}$ 照片, 可以看出, $\mathrm{BaTiO}_{3}$ 纳米 线形貌和尺寸较为均匀, 表面光滑平整呈现豆荚状, 长径比较大。对制得的 $\mathrm{Na}_{2} \mathrm{Ti}_{3} \mathrm{O}_{7}$ 纳米线、 $\mathrm{BaTiO}_{3}$ 纳米线采用 XRD 分析其物相, 如图 1(b)中 XRD 结 果所示, $\mathrm{Na}_{2} \mathrm{Ti}_{3} \mathrm{O}_{7}$ 纳米线在 $10.5^{\circ} 、 25.7^{\circ}$ 和 $29.9^{\circ}$ 均表 现出了尖锐的衍射峰, 分别对应(100)、(110)和(003) 晶面, 可以与 $\mathrm{PDF}^{\#} 31-1329$ 卡片一一对应, 表现出 了典型的单斜相; $\mathrm{BaTiO}_{3}$ 纳米线在 $22.6^{\circ} 、 31.7^{\circ}$ 、 $38.9^{\circ} 、 45.4^{\circ}$ 和 $56.3^{\circ}$ 均表现出了尖锐的衍射峰, 分别 对应(100)、(110)、(111)、(200)和(211)晶面，可以 与 $\mathrm{PDF}^{\#} 05-0626$ 卡片一一对应, 表现出了典型的四 方相。

采用透射电镜进一步观察 $\mathrm{BaTiO}_{3}$ 纳米线的形 貌。如下图 1(c) (d)所示, 分别为 $\mathrm{BaTiO}_{3}$ 纳米线的 TEM 照片、高分辨 TEM 照片及傅里叶变换图像。
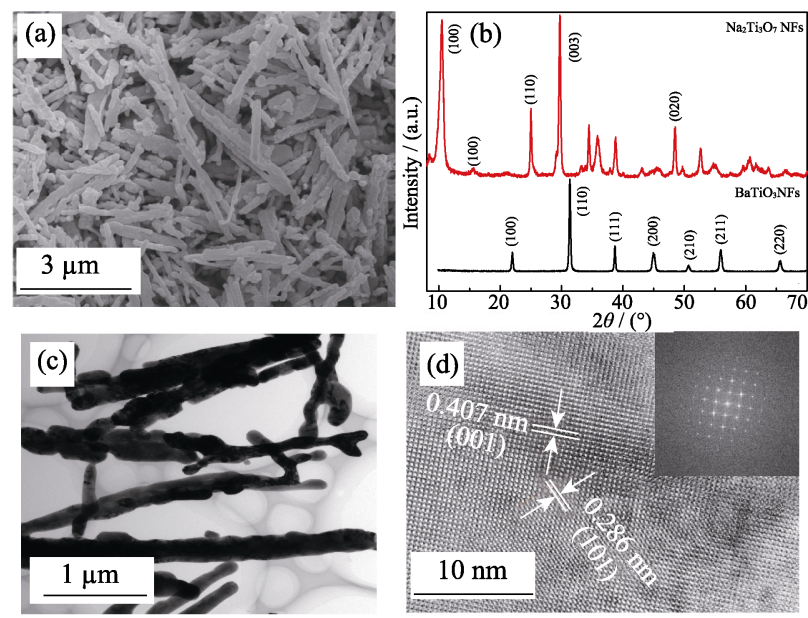

图 1 (a) $\mathrm{BaTiO}_{3}$ 纳米线的 $\mathrm{SEM}$ 照片; (b) $\mathrm{Na}_{2} \mathrm{Ti}_{3} \mathrm{O}_{7}$ 纳米线、 $\mathrm{BaTiO}_{3}$ 纳米线的 $\mathrm{XRD}$ 图谱; $\mathrm{BaTiO}_{3}$ 纳米线的(c) TEM 照片, (d)高分辨 TEM 照片及快速傅里叶变换图

Fig. 1 (a) SEM image of $\mathrm{BaTiO}_{3}$ nanofibers; (b) XRD patterns of $\mathrm{Na}_{2} \mathrm{Ti}_{3} \mathrm{O}_{7}$ and $\mathrm{BaTiO}_{3}$ nanofibers; (c) TEM image, (d) HRTEM and FFT image of $\mathrm{BaTiO}_{3}$ nanofibers
可以明显观察到 $\mathrm{BaTiO}_{3}$ 纳米线的外表面呈现一节 一节分段的豆荚状结构, 里面完整且无断裂现象。 如图 1(d)所示, 对 $\mathrm{BaTiO}_{3}$ 纳米线的晶格条纹进行标 定，其晶面间距分别为 0.407 和 $0.286 \mathrm{~nm}$, 分别对应 (001)和(101)晶面。以上证明成功合成了 $\mathrm{BaTiO}_{3}$ 纳 米线。

\section{$2.2 \mathrm{BaTiO}_{3}-\mathrm{P}(\mathrm{VDF}-\mathrm{HFP})$ 复合物微观形貌研究}

通过观察 $\mathrm{BaTiO}_{3}-\mathrm{P}(\mathrm{VDF}-\mathrm{HFP})$ 复合物膜的表面 微观形貌, 分别研究不同含量的 $\mathrm{BaTiO}_{3}$ 纳米线在聚 合物中的分布以及相容性情况，如图 2 所示。其中 图 (a) 为纯 $P(V D F-H F P)$ 膜, (b) (d) 分别为不同 $\mathrm{BaTiO}_{3}$ 纳米线含量的复合物膜表面形貌。可以很清 楚的看到, 含量为 $5 \mathrm{vol} \%$ 和 $10 \mathrm{vol} \%$ 时, $\mathrm{BaTiO}_{3}$ 纳米 线均匀地分散于聚合物基体中，结合性很好且没有 明显的团聚，但是随其含量的增加，20vol\%的复合 物膜表面出现部分可见的孔洞及团聚。这是由于 $\mathrm{BaTiO}_{3}$ 表面本身存在 $-\mathrm{OH}$ 基团, 可以很好地与 $\mathrm{P}(\mathrm{VDF}-\mathrm{HFP})$ 基体结合。因此在较低含量时, $\mathrm{BaTiO}_{3}$ 纳米线在复合物中具有良好的分散性，和聚合物的 相容性良好。

\section{3 $\mathrm{BaTiO}_{3}-\mathrm{P}(\mathrm{VDF}-\mathrm{HFP})$ 复合物介电性能研究}

图 3 分别展示了不同含量的 $\mathrm{BaTiO}_{3}-\mathrm{P}(\mathrm{VDF}-$ HFP)复合物的介电常数和介电损耗随频率变化的 规律。根据图 3(a) 可以发现，同一频率下, $\mathrm{BaTiO}_{3}-\mathrm{P}$ (VDF-HFP)复合物的介电常数随着 $\mathrm{BaTiO}_{3}$ 纳米线 含量的增加而显著增加; 含量相同时, 随着频率的 提高 $\mathrm{BaTiO}_{3}-\mathrm{P}(\mathrm{VDF}-\mathrm{HFP})$ 复合物的介电常数减小。 如: $1 \mathrm{kHz}$ 时, $5 \mathrm{vol} \% 、 10 \mathrm{vol} \%$ 和 $20 \mathrm{vol} \%$ 含量的 $\mathrm{BaTiO}_{3}-\mathrm{P}(\mathrm{VDF}-\mathrm{HFP})$ 复合物的介电常数分别为 9.09 、 18.40 和 $30.69 ; 1 \mathrm{MHz}$ 时, 三者的介电常数分别降低 至 6.79、13.64 和 23.82 。复合物的最大介电常数相
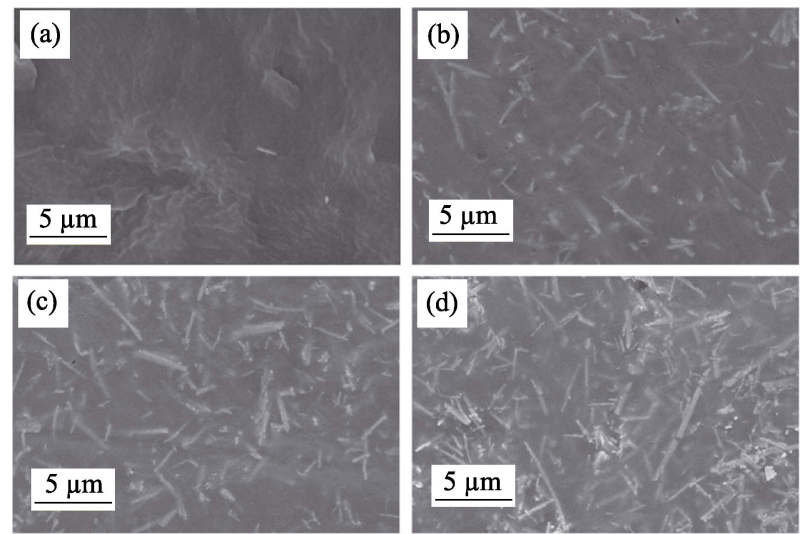

图 2 (a)纯 P(VDF-HFP)膜，(b) $5 \mathrm{vol} \%$ ，(c) $10 \mathrm{vol} \%$ 和(d) $20 \mathrm{vol} \%$ 的 $\mathrm{BaTiO}_{3}$ 纳米线复合物膜表面 SEM 照片

Fig. 2 Surface SEM images of (a) pure P(VDF-HFP) and composites filled with various $\mathrm{BaTiO}_{3}$ nanofibers: (b) $5 \mathrm{vol} \%$; (c) $10 \mathrm{vol} \%$; (d) $20 \mathrm{vol} \%$ 

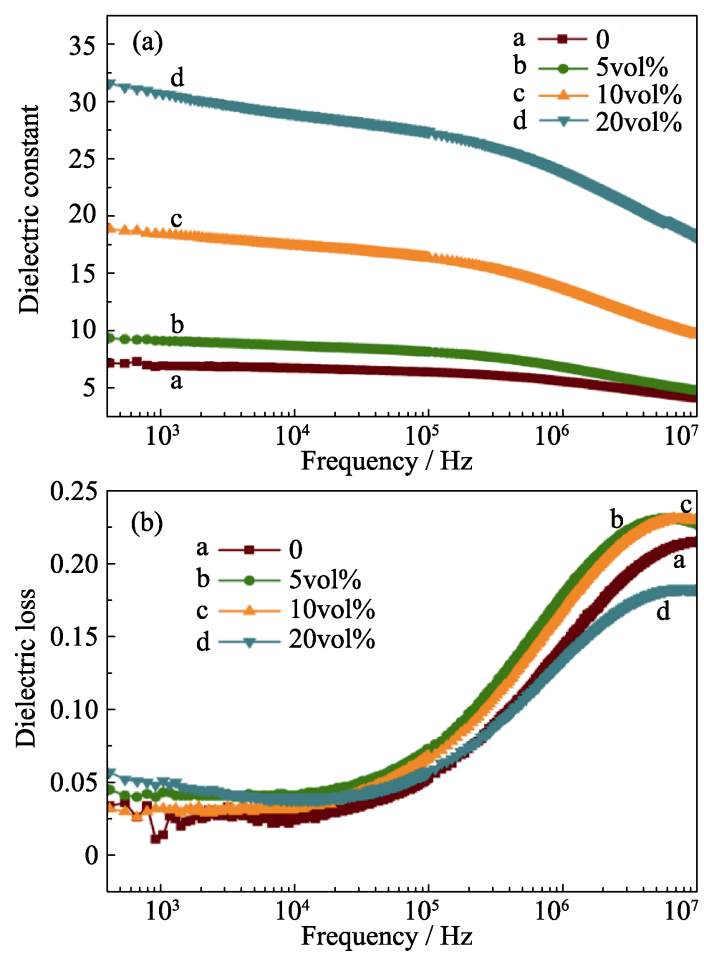

图 $3 \mathrm{BaTiO}_{3}$ 纳米线复合物的(a)介电常数和(b)介电损耗随 频率变化规律

Fig. 3 (a) Dielectric constant of $\mathrm{BaTiO}_{3}$-P (VDF-HFP) and (b) dielectric loss of $\mathrm{BaTiO}_{3}-\mathrm{P}$ (VDF-HFP) composites as a function of frequency

当于纯 $\mathrm{P}(\mathrm{VDF}-\mathrm{HFP})$ 的四倍, 除了 $\mathrm{BaTiO}_{3}$ 纳米线本 身具有较大的介电常数外, 还要归因于陶瓷与聚合 物之间的界面效应 ${ }^{[22]}$ 。根据图 3(b) 可以发现, 同一 含量的 $\mathrm{BaTiO}_{3}-\mathrm{P}(\mathrm{VDF}-\mathrm{HFP})$ 复合物, 其介电损耗随 着频率的增加先保持稳定而后增加, 且保持在较小 的范围，最大不超过 0.25 。

\section{$2.4 \mathrm{BaTiO}_{3}-\mathrm{P}(\mathrm{VDF}-\mathrm{HFP})$ 复合物储能特性研究}

采用 Weibull 统计分析方法衡量复合材料抗击 穿电场值的可靠性，方法如下：每个组分的复合物 材料分别测试 9 个抗击穿电场值, 将其按照升序进 行排列 $E_{1}<E_{2}<E_{3}<\cdots \cdots<E_{\mathrm{n}}$, 根据公式:

$$
P=1-\exp \left[-\left(\frac{E}{E_{0}}\right)^{\beta}\right]
$$

其中 $E$ 为实验测得的抗击穿强度, $P$ 为累积击穿概 率, $E_{0}$ 为 Weibull 函数的特征击穿强度 (样品在击穿 概率为 $63.2 \%$ 时的击穿强度)。 $\beta$ 表示样品击穿强度 的分布情况, 值越大, 说明样品击穿强度的分布越 窄，可靠性越好。

根据 IEEE 930-2004 标准公布的方法, 每个样 品的击穿概率如公式:

$$
P_{i}=\frac{i-0.44}{n+0.25}
$$

其中, $i$ 为测量样品数据的序号, $n$ 为测试样品总数。
其相应参数如表 1 所示。图 4(a)为通过拟合获得的 Weibull 分布曲线, 其中纵坐标为 $\mathrm{BaTiO}_{3}-\mathrm{P}$ (VDF-HFP)复合材料失效概率(击穿概率), 横坐标 为其抗击穿电场值的大小。

除了要求具有高的介电常数以外, 高的抗击穿 电场是提高储能密度的关键因素。从图 4(b)可以看 出, 纯聚合物基体具有优异的绝缘性, 其抗击穿电 场大小为 $397 \mathrm{kV} / \mathrm{mm}$, 随着 $\mathrm{BaTiO}_{3}$ 纳米线含量的增 加, 其抗击穿点明显降低, 三者的抗击穿电场大小 依次为 $244 、 198$ 和 $72 \mathrm{kV} / \mathrm{mm}$ 。这是由于陶瓷相与 基体相介电常数的巨大差异使得电场在复合物内部 分布不均匀, 另外, 不可避免的团聚和缺陷也会降 低材料的绝缘性。

表 1 不同 $\mathrm{BaTiO}_{3}$ 纳米线填充量的复合物拟合 获得抗击穿电场的 Weibull 统计分析参数

Table 1 Weibull distribution of the dielectric breakdown strength of composites filled with various $\mathrm{BaTiO}_{3}$ nanofibers

\begin{tabular}{ccc}
\hline Contents of $\mathrm{BaTiO}_{3}$ nanofibers & $\beta$ & $E_{0} /\left(\mathrm{kV} \cdot \mathrm{mm}^{-1}\right)$ \\
\hline 0 & 8.24 & 397 \\
$5 \mathrm{vol} \%$ & 6.16 & 244 \\
$10 \mathrm{vol} \%$ & 5.46 & 198 \\
$20 \mathrm{vol} \%$ & 2.51 & 72 \\
\hline
\end{tabular}
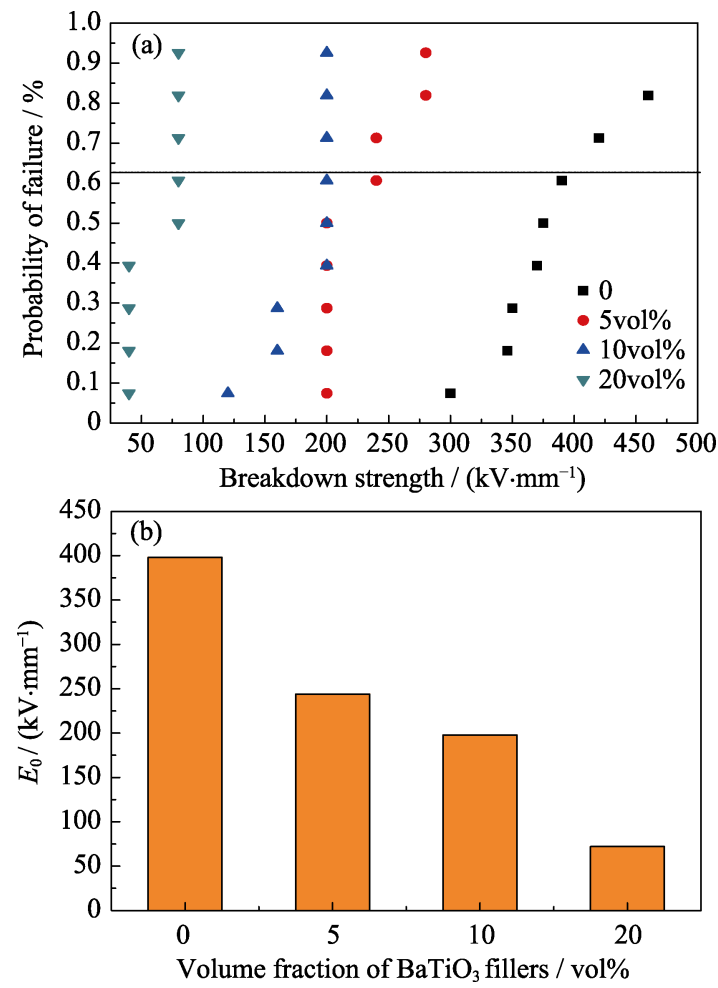

图 $4 \mathrm{BaTiO}_{3}$ 纳米线的 $\mathrm{P}(\mathrm{VDF}-\mathrm{HFP})$ 复合物与纯 $\mathrm{P}(\mathrm{VDF}-\mathrm{HFP})$ 的抗击穿电场

Fig. 4 Breakdown strength of $\mathrm{BaTiO}_{3}-\mathrm{P}(\mathrm{VDF}-\mathrm{HFP})$ composite and pure $\mathrm{P}$ (VDF-HFP) 
根据线性介电材料储能密度的计算公式,

$$
U=\frac{1}{2} \varepsilon_{0} \varepsilon_{r} E^{2}
$$

其中, $U$ 为能量密度 $\left(\mathrm{J} / \mathrm{cm}^{3}\right), E$ 为应用的电场强度 $(\mathrm{kV} / \mathrm{mm}), \varepsilon_{0}$ 为真空介电常数 $\left(8.84 \times 10^{-12} \mathrm{~F} / \mathrm{m}\right), \varepsilon_{r}$ 为相对介电常数。可以看出在固定的电场条件下电 位移与复合物的相对介电常数成正比，此前图 3(a) 讨论了复合物介电常数与 $\mathrm{BaTiO}_{3}$ 纳米线含量的关 系，因此电位移随着 $\mathrm{BaTiO}_{3}$ 纳米线含量的增加而增 大是由于对应的介电常数有所增加。同时, 介电材 料储能密度的大小除了跟介电常数相关外, 还与材 料的抗击穿电场直接相关。且抗击穿电场为二次方 指数项，因此提高材料的抗击穿电场能更有效地提 高材料的储能密度。

不同含量的 $\mathrm{BaTiO}_{3}$ 纳米线与 $\mathrm{P}(\mathrm{VDF}-\mathrm{HFP})$ 复合 物的 D-E loops 如图 5(a) (c) 所示, 其含量分别为 $5 \mathrm{vol} \% 、 10 \mathrm{vol} \%$ 以及 $20 \mathrm{vol} \%$ 。根据公式 3 , 可以得 出三种不同含量聚合物的最大放电能量密度分别为 $2.58 、 1.14 、 0.11 \mathrm{~J} / \mathrm{cm}^{3}$, 同时, 三者的最大能量储存 密度分别为 $4.89 、 1.73$ 和 $0.57 \mathrm{~J} / \mathrm{cm}^{3}$, 对应的抗击穿 电场皆为该含量下的最大场强。因此, 随着 $\mathrm{BaTiO}_{3}$ 纳米线体积分数的增加, 复合物的最大放电能量密 度与能量储存密度均减小。根据能量密度的计算公 式(3)可知, 抗击穿电场值大小对能量密度的影响更
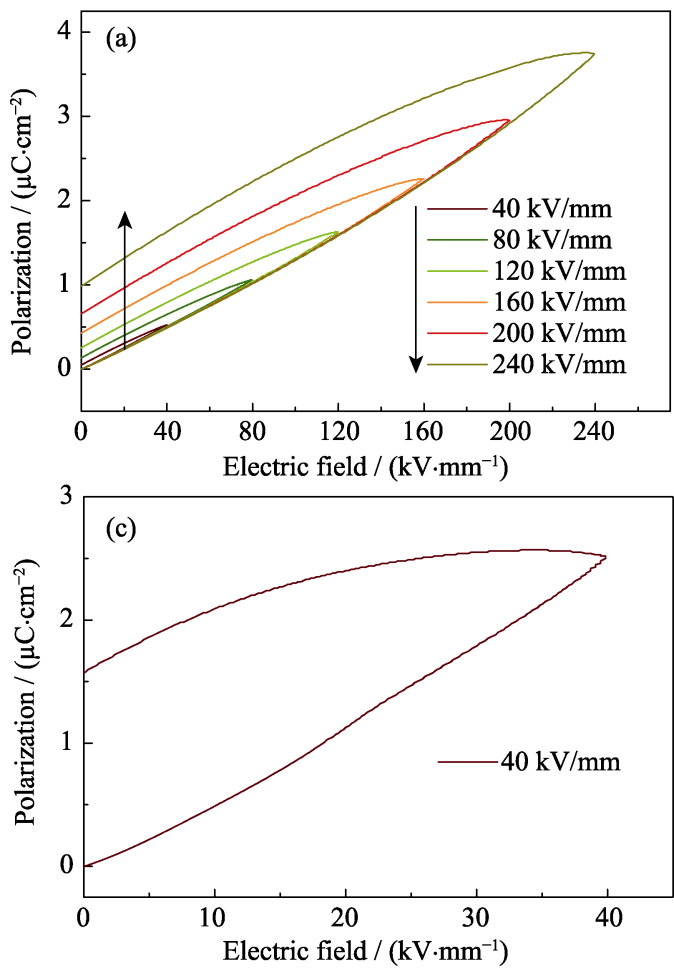

大，因而随着 $\mathrm{BaTiO}_{3}$ 纳米线体积分数的增加其抗击 穿电场值减小, 虽然介电常数增大, 但能量密度值 依然递减。研究表明, 纯 P(VDF-HFP)聚合物的最大 能量储存密度为 $4.06 \mathrm{~J} / \mathrm{cm}^{3}$, 添加了 $\mathrm{BaTiO}_{3}$ 纳米线 后, 与纯有机物的能量密度相比, 复合物的最大能 量密度提高了 $20 \%{ }^{[13]}$ 。

\section{3 结论}

1) 通过两步水热法成功制备了具有四方相，豆 荚状微观形貌的 $\mathrm{BaTiO}_{3}$ 纳米线。

2) $\mathrm{BaTiO}_{3}-\mathrm{P}(\mathrm{VDF}-\mathrm{HFP})$ 复合物中, $\mathrm{BaTiO}_{3}$ 含量 为 $5 \mathrm{vol} \%$ 时，纳米线均匀地分散于聚合物基体中， 结合性很好且没有明显的团聚，但当 $\mathrm{BaTiO}_{3}$ 纳米线 含量增加至 $20 \mathrm{vol} \%$ 时, 二者的结合性变差, 出现孔 洞及团聚现象。因此，含量较高时二者的相容性有 待进一步提高。

3) 随着 $\mathrm{BaTiO}_{3}$ 纳米线含量的增加, $\mathrm{BaTiO}_{3}-\mathrm{P}$ (VDF-HFP)复合物的介电常数增大, $1 \mathrm{kHz}$ 时, 该体 系最大介电常数为 30.69 , 介电损耗略有增大但基 本维持在较小的数值 $(<0.25)$; 复合物的抗击穿电场 随着 $\mathrm{BaTiO}_{3}$ 纳米线含量的增加而减小; 填料含量为 $5 \mathrm{vol} \%$ 的复合物在电场强度为 $240 \mathrm{kV} / \mathrm{mm}$ 时, 放电 能量密度取得最大值 $2.58 \mathrm{~J} / \mathrm{cm}^{3}$, 含量低且储能密 度大最适宜制备储能材料。
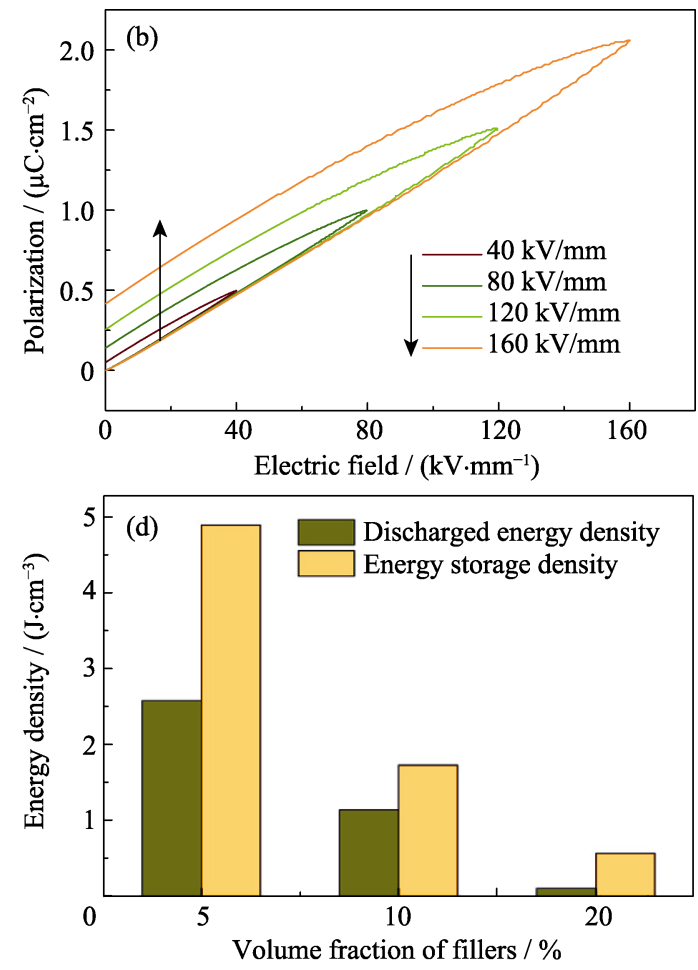

图 5 (a) $5 \mathrm{vol} \%$, (b) $10 \mathrm{vol} \%$ 和(c) $20 \mathrm{vol} \% \mathrm{BaTiO}_{3}$ 纳米线复合物膜的电位移一电场曲线及其(d)能量密度

Fig. 5 Displacement hysteresis loops of composites filled with various $\mathrm{BaTiO}_{3}$ nanofibers (a) $5 \mathrm{vol} \%$, (b) $10 \mathrm{vol} \%$, (c) $20 \mathrm{vol} \%$ and (d) energy density of composites 
参考文献:

[1] DANG Z M, YUAN J K, YAO S H, et al. Flexible nanodielectric materials with high permittivity for power energy storage. $A d$ vanced Materials, 2013, 25(44): 6334-6365.

[2] YAO L M, PAN Z B, LIU S H, et al. Significantly enhanced energy density in nanocomposite capacitors combining the $\mathrm{TiO}_{2}$ nanorod array with poly(vinylidene fluoride). ACS Applied Materials \& Interfaces, 2016, 8(39): 26343-26351.

[3] SHEN Y, SHEN D S, ZHANG X, et al. High energy density of polymer nanocomposites at a low electric field induced by modulation of their topological-structure. Journal of Materials Chemis$\operatorname{try} A, 2016, \mathbf{4}(21): 8359-8365$.

[4] WANG C Y, CHEN W T, XU C, et al. Fluorinated polyimide/ POSS hybrid polymers with high solubility and low dielectric constant. Chinese Journal of Polymer Science, 2016, 34(11): 13631372.

[5] ZIA T H, KHAN A N, HUSSAIN M, et al. Enhancing dielectric and mechanical behaviors of hybrid polymer nanocomposites based on polystyrene, polyaniline and carbon nanotubes coated with polyaniline. Chinese Journal of Polymer Science, 2016, 34(12): 1500-1509.

[6] ZHANG X, SHEN Y, ZHANG Q, et al. Ultrahigh energy density of polymer nanocomposites containing $\mathrm{BaTiO}_{3} @ \mathrm{TiO}_{2}$ nanofibers by atomic-scale interface engineering. Advanced Materials, 2015, 27(5): 819-824.

[7] XIE L, HUANG X, YANG $\mathrm{K}$, et al. "Grafting to" route to PVDF-HFP-GMA/BaTiO 3 nanocomposites with high dielectric constant and high thermal conductivity for energy storage and thermal management applications. Journal of Materials Chemistry A, 2014, 2(15): 5244-5251.

[8] TANG H X, LIN Y R, SODANO H A. Nanocomposite capacitors: enhanced energy storage in nanocomposite capacitors through aligned PZT nanowires by uniaxial strain assembly. Advanced Energy Materials, 2012, 2(4): 469-476.

[9] LUO H, ROSCOW J, ZHOU X F, et al. Ultra-high discharged energy density capacitor using high aspect ratio $\mathrm{Na}_{0.5} \mathrm{Bi}_{0.5} \mathrm{TiO}_{3}$ nanofibers. Journal of Materials Chemistry A, 2017, 5(15): 7091-7102.

[10] XIE L, HUANG X, HUANG Y, et al. Core-shell structured hyperbranched aromatic polyamide/ $\mathrm{BaTiO}_{3}$ hybrid filler for poly (vinylidene fluoride-trifluoroethylene-chlorofluoroethylene) nanocomposites with the dielectric constant comparable to that of percolative composites. ACS Applied Materials \& Interfaces, 2013, 5(5): $1747-1756$.

[11] HU P H, SONG Y, LIU H Y, et al. Largely enhanced energy den- sity in flexible $\mathrm{P}(\mathrm{VDF}-\mathrm{TrFE})$ nanocomposites by surface-modified electrospun $\mathrm{BaSrTiO}_{3}$ fibers. Journal of Materials Chemistry A, 2013, 1(5): 1688-1693.

[12] BOWEN C P, NEWNHAM R E, RANDALL C A. Dielectric properties of dielectrophoretically assembled particulate-polymer composites. Journal of Materials Research, 1998, 13(1): 205-210.

[13] LUO H, ZHANG D, JIANG C, et al. Improved dielectric properties and energy storage density of poly(vinylidene fluoride-cohexafluoropropylene) nanocomposite with hydantoin epoxy resin coated $\mathrm{BaTiO}_{3}$. ACS Applied Materials \& Interfaces, 2015, 7(15): 8061-8069.

[14] YANG Y, SUN H L, ZHU B P, et al. Enhanced dielectric performance of three phase percolative composites based on thermoplasticceramic composites and surface modified carbon nanotube. Applied Physics Letters, 2015, 106(1): 012902.

[15] ZHAN J Y, TIAN G F, WU Z P, et al. Preparation of polyimide/ $\mathrm{BaTiO}_{3} / \mathrm{Ag}$ nanocomposite films via in situ technique and study of their dielectric behavior. Chinese Journal of Polymer Science, 2014, 32(4): 424-431.

[16] PRATEEK, THAKUR V K, GUPTA R K. Recent progress on ferroelectric polymer-based nanocomposites for high energy density capacitors: synthesis, dielectric properties, and future aspects. Chemical Reviews, 2016, 116(7): 4260-4317.

[17] TANG H X, LIN Y R, SADANO H A. Synthesis of high aspect ratio $\mathrm{BaTiO}_{3}$ nanowires for high energy density nanocomposite capacitors. Advanced Energy Materials, 2013, 3(4): 451-456.

[18] RAHIMABADY M, MIRSHEKARLOO M S, YAO K, et al. Dielectric behaviors and high energy storage density of nanocomposites with core-shell $\mathrm{BaTiO}_{3} @ \mathrm{TiO}_{2}$ in poly(vinylidene fluoridehexafluoropropylene). Physical Chemistry Chemical Physics, 2013, 15(38): 16242-16248.

[19] TANG H X, LIN Y R, ANDREWS C, et al. Nanocomposites with increased energy density through high aspect ratio PZT nanowires. Nanotechnology, 2011, 22(1): 15702-15709.

[20] PAN Z B, YAO L M, ZHAI J W, et al. High-energy-density polymer nanocomposites composed of newly structured one-dimensional $\mathrm{BaTiO}_{3} @ \mathrm{Al}_{2} \mathrm{O}_{3}$ nanofibers. ACS Applied Materials \& Interfaces, 2017, 9(4): 4024-4033.

[21] TANG H X, SODANO H A. Ultra high energy density nanocomposite capacitors with fast discharge using $\mathrm{Ba}_{0.2} \mathrm{Sr}_{0.8} \mathrm{TiO}_{3}$ nanowires. Nano Letters, 2013, 13(4): 1373-1379.

[22] WANG Y, CUI J, YUAN Q, et al. Significantly enhanced breakdown strength and energy density in sandwich-structured barium titanate/poly(vinylidene fluoride) nanocomposites. Advanced Materials, 2015, 27(42): 6658-6663. 